\title{
A Jamming-Based MAC Protocol for Wireless Multihop Ad Hoc Networks
}

\author{
Shiang-Rung Ye, You-Chiun Wang, and Yu-Chee Tseng \\ Department of Computer Science and Information Engineering \\ National Chiao Tung University \\ Hsin-Chu, 30010, Taiwan \\ Email: $\{$ shiarung,wangyc, yctseng $\} @$ csie.nctu.edu.tw
}

\begin{abstract}
-
One critical issue in multihop ad hoc networks is the medium access control (MAC). The IEEE 802.11 MAC protocol is originally designed for fully connected, one-hop ad hoc networks but not for multihop ad hoc networks. In addition to the well known hiddenterminal problem, we found that IEEE 802.11 also suffers from an erroneous reservation problem which occurs when RTS-CTS exchange fails but the channel is incorrectly reserved. In this paper, we propose a jamming-based MAC (JMAC) protocol that is not only free from both hidden-terminal and the erroneous reservation problems but also allows more concurrent transmission/reception activities for stations within each other's transmission range. The idea behind the JMAC is to separate source stations' traffic from destination stations' traffic into different channels (i.e., dividing the shared medium into two channels), and explicitly signal the channel status by jamming the channels. Simulation results show that the JMAC can provide higher channel utilization when system is in high traffic load and the frame size is medium or large.
\end{abstract}

Keywords: IEEE 802.11, medium access control (MAC), mobile ad hoc network, mobile computing, protocol, wireless communication.

\section{INTRODUCTION}

The ad hoc network is a spontaneous network that consists solely of mobile stations without base stations. It can be applied in many contexts such as military communication, disaster rescue, and outdoor/indoor due to its features of convenicence in deployment and flexibility in reconfiguration. Depending on its configuration, an ad hoc network can be classified as fully connected or multihop. In this work, we focus on multihop ad hoc networks

One of the issues in a multihop ad hoc network is the design of medium access control (MAC). Traditional MAC protocols such as ALOHA [1] and CSMA [2] all suffer from the wellknown hidden-terminal problem. The hidden-terminal problem occurs when some stations are hidden from the source stations and transmit while their neighbors are receiving data. In the literature, many methods have been proposed to solve the problem [3] [4] [5] [6] [7] [8] [9] [10] [11] [12]. The BTMA [3] first proposed using busy tone to avoid the hidden-terminal problem

This work is co-sponsored by the MOE Program for Promoting Academic Excellence of Universities under grant numbers A-91-H-FA07-1-4 and 89-EFA04-1-4. in an infrastructure network. In the BTMA, the whole bandwidth is divided into a data channel and a busy-tone channel. When a base station detects carrier on the data channel, it transmits busy-tone signal (sine wave) to indicate the busy state of the data channel. Any mobile station that detects the busy-tone signal will defer its transmission until the end of the busy tone. The disadvantages of using busy tone is the cost of bandwidth for the busy tone and the cost of detection time for busy-tone signal which is no longer negligible on a narrow-band channel and must be accounted for [3].

Based on the RTS-CTS-DATA dialogue, the DBTMA protocol [10] [11] uses two busy tones to convey channel states. It divides the communication bandwidth into a control channel, a data channel, and two busy tones $\left(B T_{t}\right.$ and $\left.B T_{r}\right)$. RTS and CTS frames are exchanged on the control channel, and if exchange succeeds, a source station will transmit its data and turn on the $B T_{t}$ busy tone to prevent neighboring stations from interference. Similarly, while a destination is waiting for or receiving data, it turns on the $B T_{r}$ to avoid possible collision.

Another branch of MAC design is based on RTS-CTS exchange. The MACA protocol [5] first introduces the RTSCTS exchange to prevent the hidden-terminal problem. In this scheme, a source station transmits a RTS frame to request transmission, and if the intended destination correctly receives the RTS frame, it responds with a CTS frame. For other stations that hear the RTS or CTS frame, they will reschedule their transmission at later time. In contrast to MACA, the MACA-BI [7] which is a receiver oriented protocol suppresses the RST part of RTS/CTS handshake by using a RTR (ready to receive) frame. The RTR is transmitted by a destination station for inviting a source station to transmit its data. The RTR frames are transmitted at a rate that matches the source station's incoming traffic according to backlog information of a source station which is piggybacked in data frames for helping the destination to predict the source station's traffic. However, this method is not suitable for networks with unpredictable traffic such as burstiness traffic.

The IEEE 802.11 standard adopts the RTS-CTS-DATA-ACK exchange sequence when the size of a data frame is larger than a threshold. In fact, in a large-scale network or in situations where the hidden-terminal problem is unavoidable, this exchange sequence is suggested to be used in most cases [13]. As in the 
MACAW, the RTS and CTS may collide in IEEE 802.11 so the hidden-terminal problem remains unavoidable (but will be reduced). Besides, in this work, we observe that IEEE 802.11 also suffers from an erroneous reservation problem, which occurs when a RTS/CTS exchange fails but the medium has been incorrectly reserved by the RTS and/or CTS frames. During this reserved period, all stations in the reserved area are suppressed from transmission even though the channel is idle.

In this paper, we propose a jamming-based MAC (JMAC) protocol that can satisfactorily solve the erroneous reservation problem and the hidden terminal problem. In addition, the proposed JMAC protocol allows more concurrent transmission pairs between hosts in a limited physical area, thus further increasing the channel utilization. The basic idea behind the JMAC is to separate source stations' traffic from destination stations' traffic into different channels, and explicitly signal the channel status by jamming the channels. Although the division of the channel incurs some cost, as shown in simulation results, the advantages of being free from the erroneous reservation and the hidden terminal problems and the benefits of more concurrent transmissions will compensate the cost when data frame size is median or large.

The rest of this paper is organized as follows. In Section II, we review the IEEE 802.11 standard and some deficiencies of it when it is applied to a multi-hop ad hoc network. The proposed JMAC protocol is presented in Section III. Simulation results are in Section IV. Conclusions are drawn in Section V

\section{Deficiencies of IEEE 802.11 In Multihop Ad Hoc NETWORKS}

The basic access method of IEEE 802.11 is the distributed coordination function (DCF). It specifies how to determine channel states and how to backoff when transmission fails. Two kinds of carrier senses are defined in the DCF: physical carrier sense and virtual carrier sense. The former is supported by the physical layer, while the latter is conducted by the MAC layer. The virtual carrier sense is carried out by the network allocation vector $(N A V)$, which is set according to the ID/Duration field of data/control frames. The ID/Duration field contains the information of future usage of the medium.

IEEE 802.11 adopts the RTS-CTS-DATA-ACK as one of frame exchange sequence. When the size of a data frame is larger than 3000 bits (a default value), this exchange sequence is recommended to be used [12]. The functions of RTS and CTS frames are twofold. First, they are used to check the channel state so as to prevent more than one station from transmitting data at the same time. Second, they contain NAV information to prevent the hidden terminal problem. However, since RTS/CTS frames may collide with other frame, the hidden terminal may still occur.

Besides, the use of RTS/CTS in 802.11 also suffers from an erroneous reservation problem, which may occur when RTS/CTS exchange fails, but the channel is reserved by the RTS/CTS frames. This inhibits neighboring stations from accessing the channel even though the channel is idle. Such "holding-whilewaiting" scenario may waste much bandwidth when the traffic

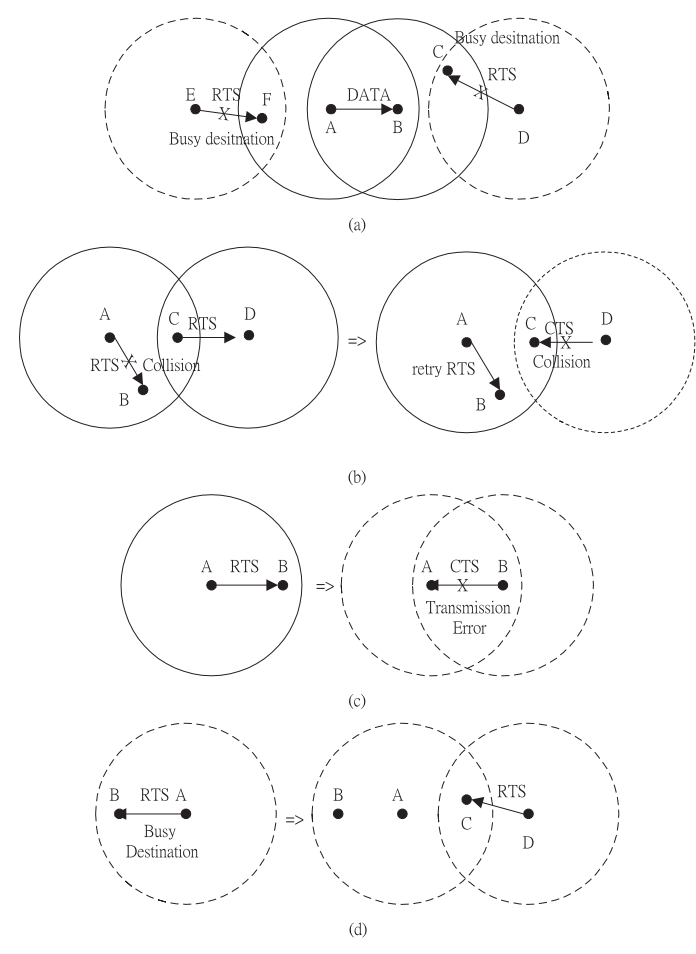

Fig. 1. Erroneous reservations caused by: (a) busy destination, (b) frame collision, (c) transmission error, and (d) the problem itself.

load is high. The erroneous reservation may occur under following conditions: busy destination, frame collision, transmission error, or the problem itself. For example, in Fig. 1(a), assume that stations $A$ and $B$ have successfully completed RTS/CTS exchange and started their transmission. In the meanwhile, if station $D$ transmits a RTS to $C$, the circle centered at $D$ will be incorrectly reserved. Similarly, if $E$ sends a RTS to $F$, the circle centered at $E$ will be incorrectly reserved too.

Frame collisions may also cause erroneous reservations. Fig. 1(b) shows that if the CTSTimeout interval is smaller than the length of a CTS frame, the erroneous reservation may also occur. In the example, $A$ and $C$ transmit RTS frames at the same time. The RTS frame from $A$ is collided with that from $C$, but the RTS frame from $C$ is successfully transmitted to $D$. While $D$ responds with a CTS frame to $C$, if $A$ retries to send a RTS frame, the CTS frame is collided at $\mathrm{C}$ and the circle centered at $D$ is erroneously reserved.

Fig. 1(c) demonstrates that transmission errors can also cause erroneous reservations. In the figure, $B$ successfully receives the RTS from $A$, but $A$ fails to receives the CTS from $B$. Then the circles centered at both $A$ and $B$ will be incorrectly reserved. It is also possible that after a station incorrectly reserves the channel, this incorrect reservation causes another reservation. In Fig. 1(d), after $A$ incorrectly reserves the channel, $D$ also incorrectly reserves the channel.

Note that the duration of an erroneous reservation may vary depending on different cases. If a RTS is received, a host will set its NAV according to the corresponding frame length. However, if the host fails to hear any frame after a timeout period, the 
NAV will be reset and the length of erroneous reservation interval would be shorten. The examples in Fig. 1(a) and (d) fall into this case. For a host hearing a CTS, the NAV can not be reset even if the host fails to receive and frame during the NAV period. This is because the host may be out of the sender's transmission range. In this case, the erroneous reservation interval would be much longer. The examples in Fig. 1(b) and (c) fall into this case.

\section{THE PRoposed MAC PRotocol}

In this section, we describe the proposed MAC protocol, $J M A C$, that is derived based on the concept of traffic separation and jamming mechanism. In JMAC, the medium is divided into two channels: $S$ channel and $R$ channel. RTS and DATA frames (source stations' traffic) are transmitted on the $S$ channel and CTS and ACK frames (destination stations's traffic) are transmitted on the $R$ channel. It is assumed that each station is equipped with two radio devices, one tuned to the $S$ channel and the other tuned to the $\mathrm{R}$ channel. The ratio of bandwidth allocated to the $R$ and $S$ channels is assumed to be $\alpha:(1-\alpha)$, where $0<\alpha<1$. How to choose an appropriate $\alpha$ will be further discussed in Section III-B.

\section{A. Protocol Behaviors}

In JMAC, a source station always transmits RTS/DATA frames on the $S$ channel but receives CTS/ACK frames on the $R$ channel. It also transmits jamming signals on the $S$ channel while waiting or receiving a CTS/ACK frame on the $R$ channel. For a destination station, while it is waiting or receiving a DATA frame on $S$ channel, it jams the $R$ channel to prevent neighboring stations from transmitting RTS frames on the $S$ channel. Jamming signal is the signal with sufficient energy causing the medium to become busy. No data is carried in jamming signal and there is no need to decode its content. The overlapping of a jamming signal and a data signal is considered as a jamming signal, and thus data signal can not be correctly recognized.

In Fig. 2, the complete access procedure of JMAC is shown between a communicating pair. Before transmitting a RTS frame on the $S$ channel, a source station senses the $R$ channel. If it is idle for a DIFS period, the source station sends a RTS on the $S$ channel, and then listens to the $R$ channel for a CTS frame. Otherwise, if the $\mathrm{R}$ channel is busy, this implies that some neighbors may be receiving data from stations that are two hops ways so the station has to perform backoff procedure. While waiting for the CTS to be sent back, the sender is required to jam the $S$ channel. The purpose of jamming the $S$ channel is similar to the reservation mechanism of RTS in 802.11, but the difference is that the medium is jammed as long as needed, depending on the result of RTS-CTS exchange. If the RTS-CTS exchange fails (indicated by a CTS_Timeout), the sender will stop jamming the $S$ channel and start the backoff procedure. On the contrary, in 802.11 , the medium is reserved for the communication period without being related to the reservation result.

After the destination station receives the RTS from the $S$ channel, it responds with a CTS frame on the $R$ channel, and

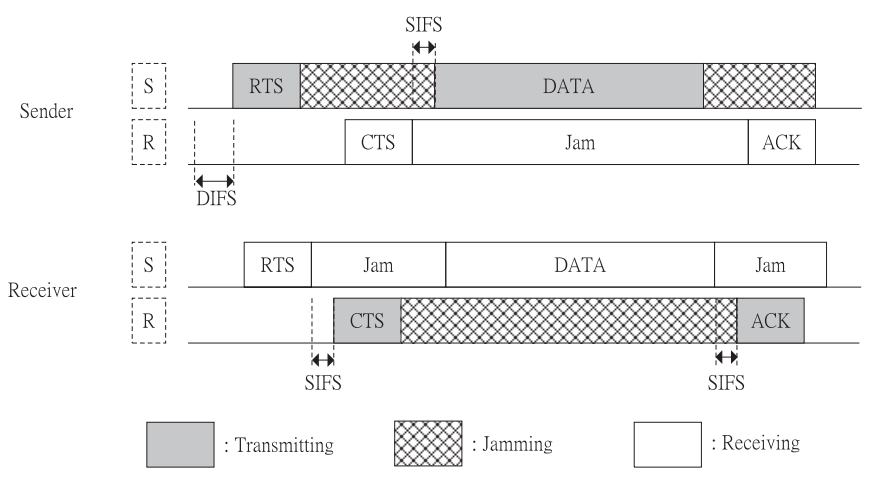

Fig. 2. The basic access procedure of the JMAC protocol.

then listens to the $S$ channel for a DATA frame. While it is waiting for the DATA frame, it also jams the $R$ channel to prevent neighboring stations from transmitting RTS frames. If unfortunately the DATA frame fails to appear after timeout, it will stop jamming the $R$ channel.

The rest of access procedure is similar. After receiving the CTS, the source transmits its DATA frame, and then jams the $S$ channel while waiting for an ACK on the $R$ channel. For the destination, after receiving the data frame successfully, it will respond with an ACK on the $R$ channel. Note that although we adopt the RTS-CTS-DATA-ACK frame exchange sequence, stations may also apply the shorted DATA-ACK sequence directly to transmit data frames. The risk is a higher penalty in case that data frames from two sources collide.

In JMAC, the backoff procedure starts after the $R$ channel becomes idle for one DIFS period and it is independent of the status of the $S$ channel. This is because the transmission of a RTS on the $S$ channel doesn't interfere with neighboring stations' reception of CTS/ACK frames on the $R$ channel, but the reception of RTS/DATA frames on the $S$ channel.

\section{B. Tuning the Factor $\alpha$}

In this section, we discuss how to choose the ratio $\alpha$ that determines the bandwidths of the $\mathrm{S}$ channel and $\mathrm{R}$ channel. Let the system transmission rate be $r$. After dividing the total bandwidth into two sub-channels, the transmission rates for the $\mathrm{S}$ channel and $\mathrm{R}$ channel are $\alpha \times r$ and $(1-\alpha) \times r$, respectively. The basic idea is to find a value of $\alpha$ such that the time of a RTS-CTSDATA-ACK exchange, denoted by $f(\alpha)$, is minimized. The $f(\alpha)$ can be expressed as follows:

$$
f(\alpha)=\frac{R T S+D A T A}{\alpha \times r}+\frac{C T S+A C K}{(1-\alpha) \times r}
$$

Differentiating $f(\alpha)$ with respect to $\alpha$, we have

$\frac{d f(\alpha)}{d \alpha}=\frac{\alpha^{2} \times(C T S+A C K)-(1-\alpha)^{2} \times(R T S+D A T A)}{\alpha^{2} \times(1-\alpha)^{2} \times r}$ 


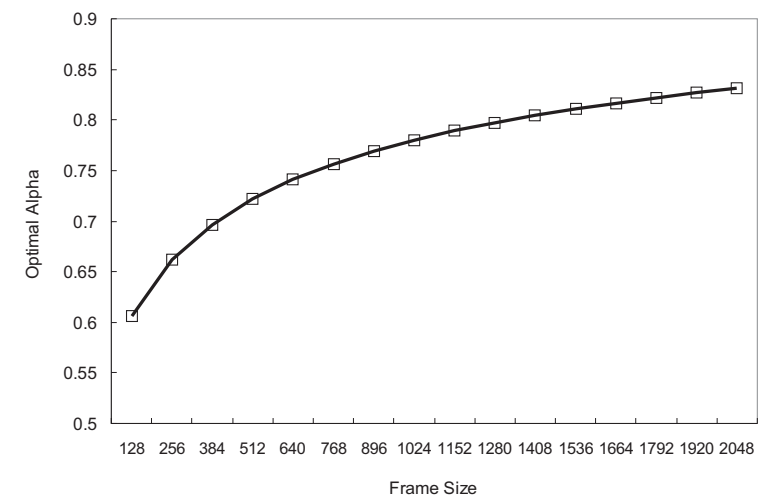

Fig. 3. Data frame size vs. the optimum alpha $\hat{\alpha}$.

Set Eq. (2) to zero, we have the optimal value $\hat{\alpha}$,

$\hat{\alpha}=\frac{R T S+D A T A \pm \sqrt{(R T S+D A T A) \times(C T S+A C K)}}{R T S+D A T A-C T S-A C K}$

Since $0<\alpha<1$,

$\hat{\alpha}=\frac{R T S+D A T A-\sqrt{(R T S+D A T A) \times(C T S+A C K)}}{R T S+D A T A-C T S-A C K}$

Although the sizes of RTS, CTS, and ACK frames are all fixed, the size of data frames may vary. Fig. 3 shows the relation of the optimum values of $\hat{\alpha}$ and the sizes of data frame sizes, $s$. The optimal $\hat{\alpha}$ tends to increase as the frame size increases. In other words, a larger data frame would require more bandwidth to be assigned to the $\mathrm{S}$ channel. There is no globally optimal $\hat{\alpha}$ for all frame sizes. So the question is : which value of $\alpha$ will be a good approximation. In Fig. 4, we plot the frame exchange time of IEEE 802.11 and JMAC when different data frame sizes and different $\alpha$ values are used. Note that for JMAC, the curve of $f$ (optimal) represents the ideal case where the datat frame size is always known and we can always choose the best $\hat{\alpha}$ to minimize the frame exchange time. As can be seen, $\alpha=0.7 \sim 0.8$ is a good approximation of the curve of $f$ (optimal) when the frame size falls in the range of $128 \sim 2048$ bytes.

The frame-exchange time of RTS-CTS-DATA-ACK in 802.11 is also shown in Fig. 4. Since 802.11 fully utilizes channel bandwidth, it is understandable that its frame-exchange time is shorter than that of JMAC. This reflects the cost incurred due to channel division. However, the analysis is under the ideal assumption that IEEE 802.11 always successfully completes its frame exchange. As discussed earlier, IEEE 802.11 may suffer from the hidden terminal and erroneous reservation problems. Thus, such an advantage may be offset by these factors. These issues will be further investigated in the next section.

\section{Performance Evaluation}

In this section, we present our simulation results and compare the performance of JMAC to that of IEEE 802.11. Parameters in Table I, Table II, and Table III are used in the simulation. In the

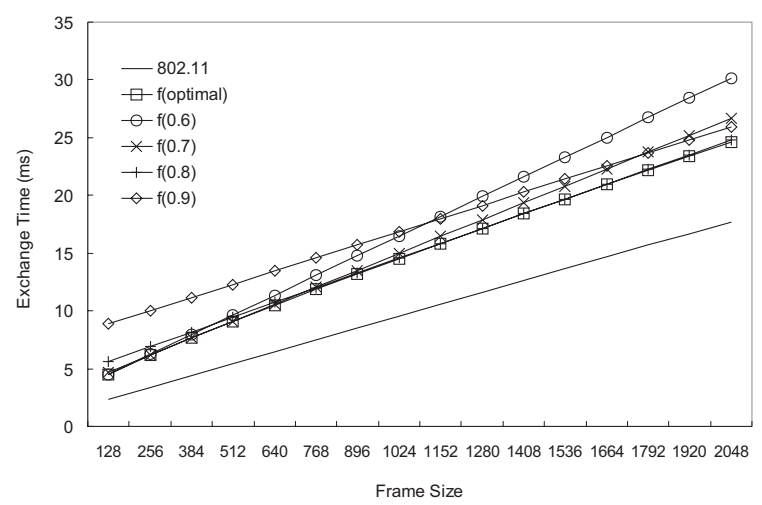

Fig. 4. Frame exchange time in IEEE 802.11 and in JMAC for different data frame sizes and different $\alpha$.

TABLE I

MAC LAYER PARAMETERS.

\begin{tabular}{|l|l|}
\hline \multicolumn{2}{|l|}{ MAC Parameters } \\
\hline Transmission Rate $\mathrm{r}$ ) & 1 (Mb/sec) \\
\hline RTS & 20 octets \\
\hline CTS & 14 octets \\
\hline ACK & 14 octets \\
\hline DATA & 1024 octets \\
\hline Retry_Count & 7 \\
\hline CWmin & 31 \\
\hline CWmax & 1023 \\
\hline CTS_Timeout & SIFS $+2 \times \tau$ \\
\hline Data_Timeout & SIFS $+2 \times \tau$ \\
\hline ACK_Timeout & SIFS $+2 \times \tau$ \\
\hline Duration/ID $($ for RTS ) & CTS + DATA + ACK $+3 \times$ aSIFSTime $+3 \times \tau$ \\
\hline Duration/ID ( for CTS ) & DATA + ACK $+2 \times$ aSIFSTime $+\tau$ \\
\hline
\end{tabular}

simulation, $N$ stations are uniformly placed in a $120 \mathrm{~m} \times 120 \mathrm{~m}$ area. The transmission range of each station is $R=30 \mathrm{~m}$ and the transmission rate is $1 \mathrm{Mbps}$. In each individual simulation run, the data frame size is assumed to be fixed. The movement of a station follows a two-state model in which each station transits from the moving state to the still state with probability $P_{S}$ and from the still state to the moving state with probability $P_{M}$. When transiting from the still to the moving state, a station chooses one of eight directions and moves in that direction with a constant speed of $1 \mathrm{~m} / \mathrm{sec}$. The total simulation time is 50 minutes in each simulation run, and frames arrive at each station according to the Possion arrival process. As to the bandwidth of the $\mathrm{S}$ channel and $\mathrm{R}$ channel, we choose $\alpha=0.78$ which is the optimal alpha for the frame size of 1024 bytes.

Figs. 5 and 6 show the aggregate throughput and mean

TABLE II

Physical LAYER PARAMETERS.

\begin{tabular}{|l|l|}
\hline \multicolumn{2}{|c|}{ DSSS PHY Specification for 2.4G Band } \\
\hline AslotTime & $20 \mu \mathrm{s}$ \\
\hline ASIFSTime & $10 \mu \mathrm{s}$ \\
\hline ADIFSTime & $50 \mu \mathrm{s}$ \\
\hline PLCP Preamble & 24 octets \\
\hline PLCP Header & 6 octets \\
\hline
\end{tabular}


TABLE III

PARAMETERS OF THE SIMULATED WIRELESS NETWORK.

\begin{tabular}{|l|l|}
\hline \multicolumn{2}{|c|}{ SimulatedEnvironment } \\
\hline Bandwidth Ratio $(\alpha)$ & 0.784 \\
\hline MaxPropagationDelay $(\tau)$ & $1(\mu \mathrm{s})$ \\
\hline Transmission Range $(\mathrm{R})$ & $30(\mathrm{~m})$ \\
\hline Simulated Area $(\mathrm{A})$ & $4 \mathrm{R}^{*} 4 \mathrm{R}\left(\mathrm{m}^{2}\right)$ \\
\hline Network Density $(\rho)$ & $3,6,9,12,15,18,21\left(\right.$ station $\left./ \pi \mathrm{R}^{2}\right)$ \\
\hline Moving Speed $(\mathrm{v})$ & $1(\mathrm{~m} / \mathrm{s})$ \\
\hline Still Probability $(\mathrm{B})$ & 0.1 \\
\hline Moving Probability $\left(\mathrm{R}_{\mathrm{M}}\right)$ & 0.9 \\
\hline
\end{tabular}

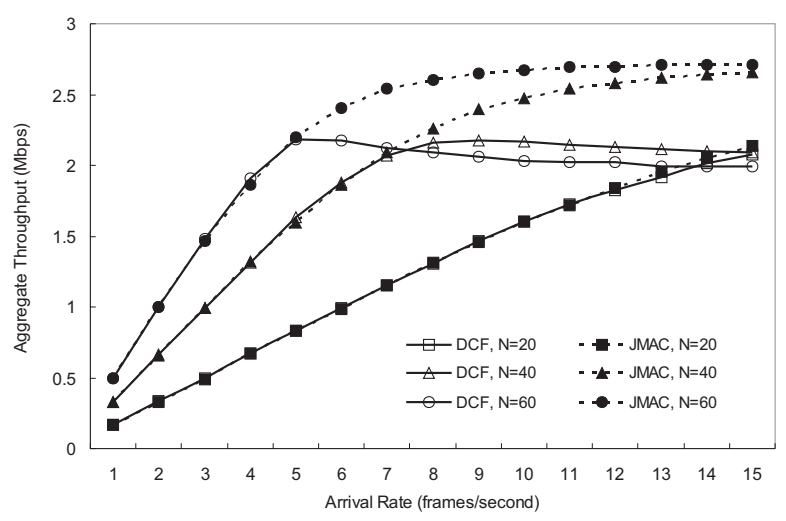

Fig. 5. Aggregate throughput versus frame arrival rate.

throughput of JMAC and IEEE 802.11 DCF under different traffic loads. At light loads (with arrival rates from 1 to 4 frames/sec), the mean throughputs of JMAC and IEEE 802.11 are very close. This is true for all values of $N$ and implies that at this stage, the throughputs are not much affected by the channel division effect for JMAC. As the traffic load increases, unless for small $N$ (such as $N=20$ ), the throughputs of JMAC will outperform the throughputs of IEEE 802.11. This implies that at this stage, the hidden terminal and erroneous reservation problems will start degrade the performances of IEEE 802.11. On the contrary, JMAC is quite resistant to such effects, and thus can still perform very well. As can be seen, after the saturation point, IEEE 802.11 will become unstable and its performance will even degrade. For JMAC, after the saturation point, JMAC can still maintain the same level of throughputs. Also note that for the case of $N=20$, the network is not saturated yet in the simulation ranges. After the saturation point (which is not shown in the figure), the similar behavior can also be observed.

\section{CONCLUSiOnS}

Besides the well known hidden terminal problem, the IEEE 802.11 also suffers from the erroneous reservation problem. In this paper, we propose a jamming-based MAC (JMAC) protocol. JMAC separates source stations' traffic from destination stations' traffic into different channels, and explicitly signals the channel status by jamming the channels. We also discuss how to choose a proper ratio for determining the bandwidths of the channels. It is shown that, the optimal ratio changes with the

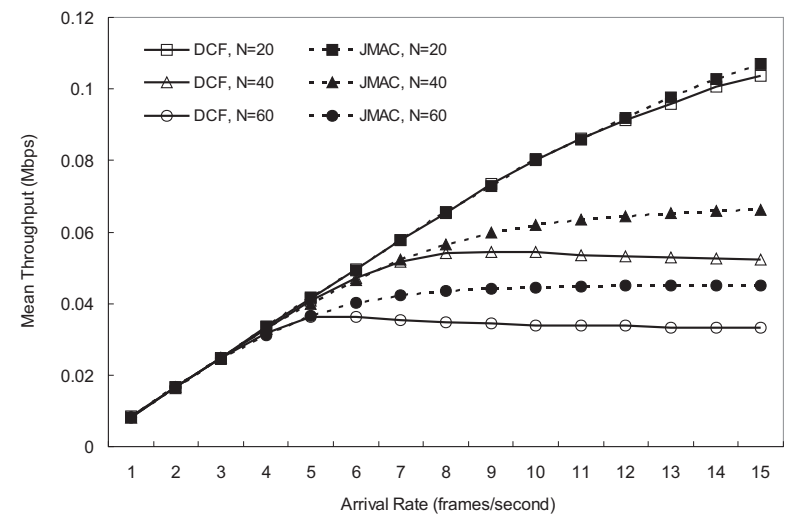

Fig. 6. Mean throughput versus frame arrival rate.

data frame size so there is no global optimal value for all sizes. But the values in the range of 0.8 and 0.7 would be a good approximation. It is also shown that channel division incurs some cost in terms of the transmission time of a RTS-CTS-DAT-ACK exchange sequence. However, our simulation results show that the advantages of being free from the erroneous reservation and the hidden terminal problems and the benefit of more concurrent transmissions of JMAC can compensate the cost of channel division and provide higher throughput compared to IEEE 802.11.

\section{REFERENCES}

[1] N. Abramson, "The Aloha System -Another Alternative for Computer communication," in AFIPS Conf. Proc., 1970, pp. 281-285.

[2] F. A. Tobagi and L. Kleinrock, "Packet switching in radio channels: Part I - carrier sense multiple-access modes and their throughput-delay characteristics," IEEE Transactions on Communication, vol. com-23, no. 12, 1975.

[3] F. A. Tobagi and L. Kleinrock, "Packet switching in radio channels: Part II - the hidden terminal problem in carrier sense multiple-access modes and the busy-tone solution," IEEE Transactions on Communication, vol. com-23, no. 12, 1975.

[4] C. Wu and V. O. K. Li, "Receiver-initiated busy-tone multiple access in packet radio networks," in ACM SIGCOMM 87 Workshop: Frontiers in Computer Communications Technology, Aug. 1987.

[5] P. Karn, "MACA - a new channel access method for packet radio," in ARRL CRRL Amateur Radio 9th Computer Networking Conference, April 1990, pp. 134-140.

[6] V. Bharghavan, A. Demers, S. Shenker, and L. Zhang, "MACAW: A media access protocol for wireless LAN's," in ACM SIGCOMM'94, 1994, pp. 212-225.

[7] F. Talucci, M. Gerla, and L. Fratta, "MACABI (MACA by invitation): A receiver oriented access protocol for wireless multiple networks," in PIMRC '97, 1994, pp. 1-4.

[8] C. L. Fullmer and J. J. Garcia-Luna-Aceves, "Floor acquisition multiple access (FAMA) for packet-radio networks," in Proc. ACM SIGCOMM 95, Aug 1995, pp. 262-273.

[9] C. L. Fullmer and J. J. Garcia-Luna-Aceves, "Solutions to hidden terminal problems in wireless networks," in IEEE ICC, 1997, pp. 55-579.

[10] J. Deng and Z. J. Haas, "Dual busy tone mulitple access (DBTMA): A new medium access control for packet radio networks," in IEEE ICUPC, 1998, pp. 973-977.

[11] Z. J. Haas and J. Deng, "Dual busy tone multiple access (DBTMA) performance evaluation," in IEEE VTC, 1999, pp. 314-319.

[12] LAN MAN Standards Committee of the IEEE Computer Society, "IEEE Std 802.11-1999, Wireless LAN Medium Access Control (MAC) and Physical Layer (PHY) specifications," IEEE, 1999.

[13] Giuseppe Bianchi, "Performance analysis of the ieee 802.11 distributed coordination function," IEEE Journal on Selected Areas in Communications, vol. 18, no. 3, 2000. 